\title{
On keen Heegaard splittings
}

\author{
Ayako Ido, Yeonhee Jang, and Tsuyoshi Kobayashi
}

\begin{abstract}
.
In this paper, we introduce a new concept of strongly keen for Heegaard splittings, and show that, for any integers $n \geq 2$ and $g \geq 3$, there exists a strongly keen Heegaard splitting of genus $g$ whose Hempel distance is $n$.
\end{abstract}

\section{$\S 1 . \quad$ Introduction}

The curve complex $\mathcal{C}(S)$ of a compact surface $S$ introduced by Harvey [4] has been used to prove many deep results in 3-dimentional topology. In particular, Hempel [5] defined the Hempel distance for a Heegaard splitting $V_{1} \cup_{S} V_{2}$ by $d(S)=d_{S}\left(\mathcal{D}\left(V_{1}\right), \mathcal{D}\left(V_{2}\right)\right)=\min \left\{d_{S}(x, y) \mid x \in \mathcal{D}\left(V_{1}\right), y \in \mathcal{D}\left(V_{2}\right)\right\}$, where $d_{S}$ is the simplicial distance of $\mathcal{C}(S)$ (for the definition, see Section 2), and $\mathcal{D}\left(V_{i}\right)$ is the disk complex of the handlebody $V_{i}(i=1,2)$. There have been many works on Hempel distance. For example, some authors showed that the existence of high distance Heegaard splittings (see 1, 3, 5, for example). Moreover, it is also shown that there exist Heegaard splittings of Hempel distance exactly $n$ for various integers $n$ (see 2, 6, 7, 11, 12, for example). Here we note that the pair $(x, y)$ in the above definition that realizes $d(S)$ may not be unique. Hence it may be natural to settle: we say that a Heegaard splitting $V_{1} \cup_{S} V_{2}$ is keen if its Hempel distance is realized by a unique pair of elements of $\mathcal{D}\left(V_{1}\right)$ and $\mathcal{D}\left(V_{2}\right)$. Namely, $V_{1} \cup_{S} V_{2}$ is keen if it satisfies the following.

- If $d_{S}(a, b)=d_{S}\left(a^{\prime}, b^{\prime}\right)=d_{S}\left(\mathcal{D}\left(V_{1}\right), \mathcal{D}\left(V_{2}\right)\right)$ for $a, a^{\prime} \in \mathcal{D}\left(V_{1}\right)$ and $b, b^{\prime} \in$ $\mathcal{D}\left(V_{2}\right)$, then $a=a^{\prime}$ and $b=b^{\prime}$.

In Proposition 3.1, we give necessary conditions for a Heegaard splitting to be keen. We note that these show that Heegaard splittings given in 6, 7, 11 are not keen (Remark 3.2). We also note that Proposition 3.1 shows that every genus-2 Heegaard splitting is not keen.

By the way, for a keen Heegaard splitting $V_{1} \cup_{S} V_{2}$, the geodesics joining the unique pair of elements of $\mathcal{D}\left(V_{1}\right)$ and $\mathcal{D}\left(V_{2}\right)$ may not be unique. In fact, Johnson 7] gives an example of a Heegaard splitting $V_{1} \cup_{S} V_{2}$ such that there is a pair of elements $(x, y)$ of $\mathcal{D}\left(V_{1}\right)$ and $\mathcal{D}\left(V_{2}\right)$ realizing the Hempel distance such that there are infinitely many geodesics joining $x$ and $y$. We say that a Heegaard splitting $V_{1} \cup_{S} V_{2}$ is strongly keen if the geodesics joining the pair of elements of $D\left(V_{1}\right)$ and $D\left(V_{2}\right)$ are unique. The main result of this paper gives the existence of strongly keen Heegaard splitting with Hempel distance $n$ for each $g \geq 3$ and $n \geq 2$ as follows.

Received Month Day, Year.

Revised Month Day, Year.

2010 Mathematics Subject Classification. 57M27.

Key words and phrases. Heegaard splitting, curve complex, distance. 
Theorem 1.1. For any integers $n \geq 2$ and $g \geq 3$, there exists a strongly keen genus-g Heegaard splitting of Hempel distance $n$.

\section{$\S 2 . \quad$ Preliminaries}

Let $S$ be a compact connected orientable surface. A simple closed curve in $S$ is essential if it does not bound a disk in $S$ and is not parallel to a component of $\partial S$. An arc properly embedded in $S$ is essential if it does not co-bound a disk in $S$ together with an $\operatorname{arc}$ on $\partial S$.

\section{Heegaard splittings}

A connected 3-manifold $C$ is a compression-body if there exists a closed (possibly empty) surface $F$ and a 0 -handle $B$ such that $C$ is obtained from $(F \times[0,1]) \cup B$ by adding 1-handles to $F \times\{1\} \cup \partial B$. The subsurface of $\partial C$ corresponding to $F \times\{0\}$ is denoted by $\partial_{-} C$, and $\partial_{+} C$ denotes the subsurface $\partial C \backslash \partial_{-} C$ of $\partial C$. A compression-body $C$ is called a handlebody if $\partial_{-} C=\emptyset$.

Let $M$ be a closed orientable 3-manifold. We say that $V_{1} \cup_{S} V_{2}$ is a Heegaard splitting of $M$ if $V_{1}$ and $V_{2}$ are handlebodies in $M$ such that $V_{1} \cup V_{2}=M$ and $V_{1} \cap V_{2}=\partial V_{1}=\partial V_{2}=S$. The genus of $S$ is called the genus of the Heegaard splitting $V_{1} \cup_{S} V_{2}$. Alternatively, given a Heegaard splitting $V_{1} \cup_{S} V_{2}$ of $M$, we may regard that there is a homeomorphism $f: \partial V_{2} \rightarrow \partial V_{1}$ such that $M$ is obtained from $V_{1}$ and $V_{2}$ by identifying $\partial V_{1}$ and $\partial V_{2}$ via $f$. When we take this viewpoint, we will denote the Heegaard splitting by the expression $V_{1} \cup_{f} V_{2}$.

\section{Curve complexes}

Let $S$ be a compact connected orientable surface with genus $g$ and $p$ boundary components. We say that $S$ is sporadic if $g=0, p \leq 4$ or $g=1, p \leq 1$. We say that $S$ is simple if $S$ contains no essential simple closed curves. We note that $S$ is simple if and only if $S$ is a 2 -sphere with at most three boundary components. The curve complex $\mathcal{C}(S)$ is defined as follows: if $S$ is non-sporadic, each vertex of $\mathcal{C}(S)$ is the isotopy class of an essential simple closed curve on $S$, and a collection of $k+1$ vertices forms a $k$-simplex of $\mathcal{C}(S)$ if they can be realized by mutually disjoint curves in $S$. In sporadic cases, we need to modify the definition of the curve complex slightly, as follows. Note that the surface $S$ is simple unless $S$ is a torus, a torus with one boundary component, or a sphere with 4 boundary components. When $S$ is a torus or a torus with one boundary component (resp. a sphere with 4 boundary components), a collection of $k+1$ vertices forms a $k$-simplex of $\mathcal{C}(S)$ if they can be realized by essential simple closed curves in $S$ which mutually intersect exactly once (resp. twice). The arc-and-curve complex $\mathcal{A C}(S)$ is defined similarly, as follows: each vertex of $\mathcal{A C}(S)$ is the isotopy class of an essential properly embedded arc or an essential simple closed curve on $S$, and a collection of $k+1$ vertices forms a $k$-simplex of $\mathcal{A C}(S)$ if they can be realized by mutually disjoint arcs or simple closed curves in $S$. The symbol $\mathcal{C}^{0}(S)$ (resp. $\mathcal{A C}^{0}(S)$ ) denotes the 0 -skeleton of $\mathcal{C}(S)$ (resp. $\mathcal{A C}(S)$ ). Throughout this paper, for a vertex $x \in \mathcal{C}^{0}(S)$ we often abuse notation and use $x$ to represent (the isotopy class of) a geometric representative of $x$.

For two vertices $a, b$ of $\mathcal{C}(S)$, we define the distance $d_{\mathcal{C}(S)}(a, b)$ between $a$ and $b$, which will be denoted by $d_{S}(a, b)$ in brief, as the minimal number of 1 -simplexes of a simplicial path in $\mathcal{C}(S)$ joining $a$ and $b$. For two subsets $A, B$ of $\mathcal{C}^{0}(S)$, we 
define $\operatorname{diam}_{S}(A, B):=$ the diameter of $A \cup B$. Similarly, we can define $d_{\mathcal{A C}(S)}(a, b)$ for $a, b \in \mathcal{A C}^{0}(S)$ and $\operatorname{diam}_{\mathcal{A C}(S)}(A, B)$ for $A, B \subset \mathcal{A C}^{0}(S)$.

For a sequence $a_{0}, a_{1}, \ldots, a_{n}$ of vertices in $\mathcal{C}(S)$ with $a_{i} \cap a_{i+1}=\emptyset(i=$ $0,1, \ldots, n-1)$, we denote by $\left[a_{0}, a_{1}, \ldots, a_{n}\right]$ the path in $\mathcal{C}(S)$ with vertices $a_{0}, a_{1}, \ldots, a_{n}$ in this order. We say that a path $\left[a_{0}, a_{1}, \ldots, a_{n}\right]$ is a geodesic if $n=d_{S}\left(a_{0}, a_{n}\right)$.

Let $C$ be a compression-body. A disk $D$ properly embedded in $C$ is essential if $\partial D$ is an essential simple closed curve in $\partial_{+} C$. Then the disk complex $\mathcal{D}(C)$ is the subset of $\mathcal{C}^{0}\left(\partial_{+} C\right)$ consisting of the vertices with representatives bounding essential disks of $C$.

For a genus- $g(\geq 2)$ Heegaard splitting $V_{1} \cup_{S} V_{2}$, the Hempel distance of $V_{1} \cup_{S} V_{2}$ is defined by $d_{S}\left(\mathcal{D}\left(V_{1}\right), \mathcal{D}\left(V_{2}\right)\right)=\min \left\{d_{S}(x, y) \mid x \in \mathcal{D}\left(V_{1}\right), y \in \mathcal{D}\left(V_{2}\right)\right\}$.

\section{Subsurface projection maps}

Let $\mathcal{P}(Y)$ denote the power set of a set $Y$. Let $S$ be a compact connected orientable surface, and let $X$ be a subsurface of $S$. We suppose that both $S$ and $X$ are non-sporadic, and each component of $\partial X$ is either contained in $\partial S$ or essential in $S$. We call the composition $\pi_{0} \circ \pi_{A}$ of maps $\pi_{A}: \mathcal{C}^{0}(S) \rightarrow \mathcal{P}\left(\mathcal{A C}^{0}(X)\right)$ and $\pi_{0}: \mathcal{P}\left(\mathcal{A C}^{0}(X)\right) \rightarrow \mathcal{P}\left(\mathcal{C}^{0}(X)\right)$ a subsurface projection if they satisfy the following: for $\alpha \in \mathcal{C}^{0}(S)$, take a representative of $\alpha$ so that $|\alpha \cap X|$ is minimal, where $|\cdot|$ is the number of connected components. Then

- $\pi_{A}(\alpha)$ is the set of all isotopy classes of the components of $\alpha \cap X$,

- $\pi_{0}\left(\left\{\alpha_{1}, \ldots, \alpha_{n}\right\}\right)$ is the union, for all $i=1, \ldots, n$, of the set of all isotopy classes of the components of $\partial N\left(\alpha_{i} \cup \partial X\right)$ which are essential in $X$, where $N\left(\alpha_{i} \cup \partial X\right)$ is a regular neighborhood of $\alpha_{i} \cup \partial X$ in $X$.

We denote the subsurface projection $\pi_{0} \circ \pi_{A}$ by $\pi_{X}$. We say that $\alpha$ misses $X$ (resp. $\alpha$ cuts $X)$ if $\alpha \cap X=\emptyset$ (resp. $\alpha \cap X \neq \emptyset)$.

Lemma 2.1. (10, Lemma 2.2]) Let $X$ be as above. Let $A$ and $B$ be subsets of $\mathcal{A C}^{0}(X)$. If $\operatorname{diam}_{\mathcal{A C}(X)}(A, B) \leq 1$, then $\operatorname{diam}_{X}\left(\pi_{0}(A), \pi_{0}(B)\right) \leq 2$.

The following lemma is proved by using the above lemma.

Lemma 2.2. (6, Lemma 2.1]) Let $X$ be as above. Let $\left[\alpha_{0}, \alpha_{1}, \ldots, \alpha_{n}\right]$ be a path in $\mathcal{C}(S)$ such that every $\alpha_{i}$ cuts $X$. Then $\operatorname{diam}_{X}\left(\pi_{X}\left(\alpha_{0}\right), \pi_{X}\left(\alpha_{n}\right)\right) \leq 2 n$.

Throughout this paper, given an embedding $\varphi: X \rightarrow Y$ between compact surfaces $X$ and $Y$, we abuse notation and use $\varphi$ to denote the map $\mathcal{C}^{0}(X) \rightarrow \mathcal{C}^{0}(Y)$ or $\mathcal{P}\left(\mathcal{C}^{0}(X)\right) \rightarrow \mathcal{P}\left(\mathcal{C}^{0}(Y)\right)$ induced by $\varphi: X \rightarrow Y$. The following two lemmas can be proved by using arguments in the proof of [6. Propositions 4.1, 4.4].

Lemma 2.3. Let $\left[\alpha_{0}, \alpha_{1}, \ldots, \alpha_{n}\right]$ and $\left[\beta_{0}, \beta_{1}, \ldots, \beta_{m}\right]$ be geodesics in $\mathcal{C}(S)$. Suppose that $\alpha_{n}$ and $\beta_{0}$ are non-separating on $S$, and let $X=\mathrm{Cl}\left(S \backslash N\left(\alpha_{n}\right)\right)$. Let $h: S \rightarrow S$ be a homeomorphism such that

- $h\left(\beta_{0}\right)=\alpha_{n}$, and

- $\operatorname{diam}_{X}\left(\pi_{X}\left(\alpha_{0}\right), \pi_{X}\left(h\left(\beta_{m}\right)\right)\right)>2(n+m)$.

Then $\left[\alpha_{0}, \alpha_{1}, \ldots, \alpha_{n}\left(=h\left(\beta_{0}\right)\right), h\left(\beta_{1}\right), \ldots, h\left(\beta_{m}\right)\right]$ is a geodesic in $\mathcal{C}(S)$.

Lemma 2.4. Let $\left[\alpha_{0}, \alpha_{1}, \ldots, \alpha_{n}\right]$ and $\left[\beta_{0}, \beta_{1}, \ldots, \beta_{m}\right]$ be geodesics in $\mathcal{C}(S)$. Suppose that $\alpha_{n-1} \cup \alpha_{n}$ and $\beta_{0} \cup \beta_{1}$ are non-separating on $S$, and let $X=\operatorname{Cl}(S \backslash$ $\left.N\left(\alpha_{n-1} \cup \alpha_{n}\right)\right)$. Let $h: S \rightarrow S$ be a homeomorphism such that

- $h\left(\beta_{0}\right)=\alpha_{n-1}, h\left(\beta_{1}\right)=\alpha_{n}$, and 
- $\operatorname{diam}_{X}\left(\pi_{X}\left(\alpha_{0}\right), \pi_{X}\left(h\left(\beta_{m}\right)\right)\right)>2(n+m-1)$.

Then $\left[\alpha_{0}, \alpha_{1}, \ldots, \alpha_{n-1}\left(=h\left(\beta_{0}\right)\right), \alpha_{n}\left(=h\left(\beta_{1}\right)\right), h\left(\beta_{2}\right), \ldots, h\left(\beta_{m}\right)\right]$ is a geodesic in $\mathcal{C}(S)$.

Remark 2.5. By the proof of [6, Propositions 4.1, 4.4], the following holds.

- Let $\left[\alpha_{0}, \alpha_{1}, \ldots, \alpha_{n}\left(=h\left(\beta_{0}\right)\right), h\left(\beta_{1}\right), \ldots, h\left(\beta_{m}\right)\right]$ be a geodesic in Lemma2.3, Then every geodesic connecting $\alpha_{0}$ and $h\left(\beta_{m}\right)$ passes through $\alpha_{n}$. In fact, for any geodesic $\left[\gamma_{0}, \gamma_{1}, \ldots, \gamma_{n+m}\right]$ in $\mathcal{C}(S)$ such that $\gamma_{0}=\alpha_{0}$ and $\gamma_{n+m}=h\left(\beta_{m}\right)$, we have $\gamma_{n}=\alpha_{n}$.

- Let $\left[\alpha_{0}, \alpha_{1}, \ldots, \alpha_{n-1}\left(=h\left(\beta_{0}\right)\right), \alpha_{n}\left(=h\left(\beta_{1}\right)\right), \ldots, h\left(\beta_{m}\right)\right]$ be a geodesic in Lemma 2.4. Then every geodesic connecting $\alpha_{0}$ and $h\left(\beta_{m}\right)$ passes through $\alpha_{n-1}$ or $\alpha_{n}$. In fact, for any geodesic $\left[\gamma_{0}, \gamma_{1}, \ldots, \gamma_{n+m-1}\right]$ in $\mathcal{C}(S)$ such that $\gamma_{0}=\alpha_{0}$ and $\gamma_{n+m-1}=h\left(\beta_{m}\right)$, we have $\gamma_{n-1}=\alpha_{n-1}$ or $\gamma_{n}=\alpha_{n}$.

\section{$\S 3 . \quad$ Keen Heegaard splittings}

Recall that a Heegaard splitting $V_{1} \cup_{S} V_{2}$ is called keen if its Hempel distance is realized by a unique pair of elements of $\mathcal{D}\left(V_{1}\right)$ and $\mathcal{D}\left(V_{2}\right)$.

Proposition 3.1. Let $V_{1} \cup_{S} V_{2}$ be a genus-g( $\left.\geq 2\right)$ Heegaard splitting with Hempel distance $n(\geq 1)$. Let $\left[l_{0}, l_{1}, \ldots, l_{n}\right]$ be a geodesic in $\mathcal{C}(S)$ such that $l_{0} \in$ $\mathcal{D}\left(V_{1}\right)$ and $l_{n} \in \mathcal{D}\left(V_{2}\right)$. If $V_{1} \cup_{S} V_{2}$ is keen, then the following holds.

(1) $l_{0}$ and $l_{n}$ are non-separating on $S$.

(2) $l_{1}$ and $l_{n-1}$ are non-separating on $S$.

(3) $l_{0} \cup l_{1}$ and $l_{n-1} \cup l_{n}$ are separating on $S$.

Proof. (1) Assume on the contrary that either $l_{0}$ or $l_{n}$ is separating on $S$. Without loss of generality, we may assume that $l_{0}$ is separating on $S$. Let $D_{0}$ be a disk properly embedded in $V_{1}$ such that $\partial D_{0}=l_{0}$. Let $V_{1}^{(1)}$ be the component of $V_{1} \backslash D_{0}$ that contains $l_{1}$, and let $V_{1}^{(2)}$ be the other component. It is easy to see that there is an essential disk $D_{0}^{\prime}$ properly embedded in $V_{1}^{(2)}$. Then $l_{0}^{\prime}:=\partial D_{0}^{\prime}$ is also disjoint from $l_{1}$, and hence, $\left[l_{0}^{\prime}, l_{1}, \ldots, l_{n}\right]$ is a geodesic in $\mathcal{C}(S)$. Hence, we have $d_{S}\left(l_{0}^{\prime}, l_{n}\right)=d_{S}\left(\mathcal{D}\left(V_{1}\right), \mathcal{D}\left(V_{2}\right)\right)$, where $l_{0}^{\prime}$ is an element of $\mathcal{D}\left(V_{1}\right)$ different from $l_{0}$, a contradiction.

(2) Assume on the contrary that either $l_{1}$ or $l_{n-1}$, say $l_{1}$, is separating on $S$. Let $S^{(1)}$ be the component of $S \backslash l_{1}$ that contains $l_{0}$. Since $l_{0}$ is non-separating on $S$ by (1) and $l_{1}$ is separating on $S$, we can see that $l_{0}$ is non-separating on $S^{(1)}$. Then there exists an essential simple closed curve $l^{*}$ on $S^{(1)}$ such that $l^{*}$ intersects $l_{0}$ transversely in one point. Let $D_{0}$ be a disk properly embedded in $V_{1}$ such that $\partial D_{0}=l_{0}$, and let $D_{0}^{+}$and $D_{0}^{-}$be the components of $\mathrm{Cl}\left(\partial N\left(D_{0}\right) \backslash \partial V_{1}\right)$, where $N\left(D_{0}\right)$ is a regular neighborhood of $D_{0}$ in $V_{1}$. Take the subarc of $l^{*}$ lying outside of the product region $N\left(D_{0}\right)$ between $D_{0}^{+}$and $D_{0}^{-}$, and let $D_{0}^{\prime \prime}$ be the disk in $V_{1}$ obtained from $D_{0}^{+} \cup D_{0}^{-}$by adding a band along the subarc of $l^{*}$. Then $l_{0}^{\prime \prime}:=\partial D_{0}^{\prime \prime}$ is also disjoint from $l_{1}$, and hence, $\left[l_{0}^{\prime \prime}, l_{1}, \ldots, l_{n}\right]$ is a geodesic in $\mathcal{C}(S)$. Hence, we have $d_{S}\left(l_{0}^{\prime \prime}, l_{n}\right)=d_{S}\left(\mathcal{D}\left(V_{1}\right), \mathcal{D}\left(V_{2}\right)\right)$, where $l_{0}^{\prime \prime}$ is an element of $\mathcal{D}\left(V_{1}\right)$ different from $l_{0}$, a contradiction.

(3) Assume on the contrary that either $l_{0} \cup l_{1}$ or $l_{n-1} \cup l_{n}$, say $l_{0} \cup l_{1}$, is nonseparating on $S$. Then there exists an essential simple closed curve $l^{*}$ on $S$ such that $l^{*}$ intersects $l_{0}$ transversely in one point and $l^{*} \cap l_{1}=\emptyset$. We can lead to a contradiction by the arguments in (2).

Q.E.D. 
Remark 3.2. (1) By Proposition [3.1] we see that every genus-2 Heegaard splitting is not keen. In fact, if a genus-2 Heegaard splitting $V_{1} \cup_{S} V_{2}$ is keen, and $\left[l_{0}, l_{1}, \ldots, l_{n}\right]$ is a path that realizes the Hempel distance, then by (1) and (2) of Proposition 3.1 we see that $l_{0} \cup l_{1}$ cuts $S$ into four punctured sphere, contradicting (3) of Proposition 3.1. Hence, if a genus- $g$ Heegaard splitting (with Hempel distance $n \geq 1$ ) is keen, then $g \geq 3$.

(2) Heegaard splittings given in 6, 7, 11] are not keen, since their Hempel distances are realized by pairs of separating elements.

\section{$\S 4$. Proof of Theorem 1.1 when $n \geq 4$}

Let $n$ and $g$ be integers with $n \geq 4$ and $g \geq 3$. Let $S$ be a closed connected orientable surface of genus $g$. Let $l_{0}$ and $l_{1}$ be non-separating simple closed curves on $S$ such that $l_{0} \cap l_{1}=\emptyset, l_{0} \cup l_{1}$ is separating and $l_{0}, l_{1}$ are not parallel on $S$. Let $F_{1}=\mathrm{Cl}\left(S \backslash N\left(l_{1}\right)\right)$. Choose and fix an integer $k \in\{2,3, \ldots, n-2\}$. Let $\left[l_{1}^{\prime}, l_{2}^{\prime}, \ldots, l_{k}^{\prime}\right]$ and $\left[l_{1}^{\prime \prime}, l_{2}^{\prime \prime}, \ldots, l_{n-k}^{\prime \prime}\right]$ be geodesics in $\mathcal{C}(S)$ such that $l_{1}^{\prime}, l_{k}^{\prime}, l_{1}^{\prime \prime}$ and $l_{n-k}^{\prime \prime}$ are non-separating on $S$. (For the existence of such geodesics, see [6] or the proof of Claim 4.14 below for example.) By [9, Proposition 4.6], there exist homeomorphisms $h_{1}: S \rightarrow S$ and $h_{2}: S \rightarrow S$ such that

- $h_{1}\left(l_{1}^{\prime}\right)=l_{1}$,

- $h_{2}\left(l_{1}^{\prime \prime}\right)=l_{1}$,

- $\operatorname{diam}_{F_{1}}\left(\pi_{F_{1}}\left(l_{0}\right), \pi_{F_{1}}\left(h_{1}\left(l_{k}^{\prime}\right)\right)\right) \geq 4 n+16$, and

- $\operatorname{diam}_{F_{1}}\left(\pi_{F_{1}}\left(l_{0}\right), \pi_{F_{1}}\left(h_{2}\left(l_{n-k}^{\prime \prime}\right)\right)\right) \geq 4 n+16$.

Note that $\pi_{F_{1}}\left(l_{0}\right)=\left\{l_{0}\right\}$ since $l_{0} \cap l_{1}=\emptyset$. By Lemma 2.3. $\left[l_{0}, l_{1}\left(=h_{1}\left(l_{1}^{\prime}\right)\right), h_{1}\left(l_{2}^{\prime}\right), \ldots, h_{1}\left(l_{k}^{\prime}\right)\right]$ and $\left[l_{0}, l_{1}\left(=h_{2}\left(l_{1}^{\prime \prime}\right)\right), h_{2}\left(l_{2}^{\prime \prime}\right), \ldots, h_{2}\left(l_{n-k}^{\prime \prime}\right)\right]$ are geodesics in $\mathcal{C}(S)$. Let $F_{k}=\mathrm{Cl}(S \backslash$ $\left.N\left(h_{1}\left(l_{k}^{\prime}\right)\right)\right)$. By [9, Proposition 4.6], there exists a homeomorphism $h_{3}: S \rightarrow S$ such that

- $h_{3}\left(h_{2}\left(l_{n-k}^{\prime \prime}\right)\right)=h_{1}\left(l_{k}^{\prime}\right)$, and

- $\operatorname{diam}_{F_{k}}\left(\pi_{F_{k}}\left(l_{0}\right), \pi_{F_{k}}\left(h_{3}\left(l_{0}\right)\right)\right)>2 n$.

Let $l_{i}=h_{1}\left(l_{i}^{\prime}\right)$ for $i \in\{2, \ldots, k\}, l_{i}=h_{3}\left(h_{2}\left(l_{n-i}^{\prime \prime}\right)\right)$ for $i \in\{k+1, \ldots, n-1\}$, and $l_{n}=h_{3}\left(l_{0}\right)$. By Lemma $2.3\left[l_{0}, l_{1}, \ldots, l_{n}\right]$ is a geodesic in $\mathcal{C}(S)$. Moreover, by the construction of the geodesic, the following are satisfied.

(G1) $l_{0}, l_{1}, l_{n-1}$ and $l_{n}$ are non-separating on $S$,

(G2) $l_{0} \cup l_{1}$ and $l_{n-1} \cup l_{n}$ are separating on $S$,

(G3) $\operatorname{diam}_{F_{1}}\left(\pi_{F_{1}}\left(l_{0}\right), \pi_{F_{1}}\left(l_{k}\right)\right) \geq 4 n+16$,

(G4) $\operatorname{diam}_{F_{n-1}}\left(\pi_{F_{n-1}}\left(l_{k}\right), \pi_{F_{n-1}}\left(l_{n}\right)\right) \geq 4 n+16$, and

(G5) $\operatorname{diam}_{F_{k}}\left(\pi_{F_{k}}\left(l_{0}\right), \pi_{F_{k}}\left(l_{n}\right)\right)>2 n$,

where $F_{n-1}=\mathrm{Cl}\left(S \backslash N\left(l_{n-1}\right)\right)$.

Let $C_{1}$ and $C_{2}$ be copies of the compression-body obtained by adding a 1handle to $F \times[0,1]$, where $F$ is a closed orientable surface of genus $g-1$. Let $D_{1}$ (resp. $D_{2}$ ) be the non-separating essential disk properly embedded in $C_{1}$ (resp. $C_{2}$ ) corresponding to the co-core of the 1-handle. We may assume that $\partial_{+} C_{1}=S$ and $\partial D_{1}=l_{0}$. Choose a homeomorphism $f: \partial_{+} C_{2} \rightarrow \partial_{+} C_{1}$ such that $f\left(\partial D_{2}\right)=l_{n}$.

Let $H_{1}$ and $H_{2}$ be copies of the handlebody of genus $g-1$. In the remainder of this section, we identify $\partial H_{i}$ and $\partial_{-} C_{i}(i=1,2)$ so that we obtain a keen Heegaard splitting of genus $g$ whose Hempel distance is $n$.

For each $i=1,2$, let $C_{i}^{\prime}=\mathrm{Cl}\left(C_{i} \backslash N\left(D_{i}\right)\right)$ and $X_{i}=\partial C_{i}^{\prime} \cap \partial_{+} C_{i}$. Note that $C_{i}^{\prime}$ is homeomorphic to $\partial_{-} C_{i} \times[0,1]$. Let $\varphi_{i}: C_{i}^{\prime} \rightarrow \partial_{-} C_{i} \times[0,1]$ be a 
homeomorphism such that $\varphi_{i}\left(\partial C_{i}^{\prime} \backslash \partial_{-} C_{i}\right)=\partial_{-} C_{i} \times\{1\}$ and $\varphi_{i}\left(\partial_{-} C_{i}\right)=\partial_{-} C_{i} \times\{0\}$, and let $\psi_{i}: \partial_{-} C_{i} \times\{1\} \rightarrow \partial_{-} C_{i} \times\{0\}$ be the natural homeomorphism. Let $P_{i}: X_{i} \rightarrow \partial_{-} C_{i}$ be the composition of the inclusion map $X_{i} \rightarrow \partial C_{i}^{\prime} \backslash \partial_{-} C_{i}$ and the $\operatorname{map}\left(\left.\varphi_{i}\right|_{\partial_{-} C_{i}}\right)^{-1} \circ \psi_{i} \circ\left(\left.\varphi_{i}\right|_{\partial C_{i}^{\prime} \backslash \partial_{-} C_{i}}\right): \partial C_{i}^{\prime} \backslash \partial_{-} C_{i} \rightarrow \partial_{-} C_{i}$.

It is clear that $l_{1}$ represents an essential simple closed curve on $X_{1}$. Since $l_{1}$ is non-separating on $S, P_{1}\left(l_{1}\right)$ is an essential simple closed curve on $\partial_{-} C_{1}$. By [1], there exists a homeomorphism $f_{1}: \partial H_{1} \rightarrow \partial_{-} C_{1}$ such that

$$
d_{\partial_{-} C_{1}}\left(f_{1}\left(\mathcal{D}\left(H_{1}\right)\right), P_{1}\left(l_{1}\right)\right) \geq 2 .
$$

Let $V_{1}=C_{1} \cup_{f_{1}} H_{1}$, that is, $V_{1}$ is the manifold obtained from $C_{1}$ and $H_{1}$ by identifying $\partial_{-} C_{1}$ and $\partial H_{1}$ via $f_{1}$. Note that $V_{1}$ is a handlebody.

Claim 4.1. $l_{1}$ intersects every element of $\mathcal{D}\left(V_{1}\right) \backslash\left\{l_{0}\right\}$.

Proof. Assume on the contrary that there exists an element $a$ of $\mathcal{D}\left(V_{1}\right) \backslash\left\{l_{0}\right\}$ such that $a \cap l_{1}=\emptyset$. Let $D_{a}$ be a disk in $V_{1}$ bounded by $a$, and recall that $l_{0}$ bounds the disk $D_{1}$ in $C_{1}$, and hence, in $V_{1}$ (see Fig. 1). We may assume that $\left|D_{a} \cap D_{1}\right|=\left|D_{a} \cap N\left(D_{1}\right)\right|$ and is minimal. By using innermost disk arguments, we see that $D_{a} \cap D_{1}$ has no loop components. Let $\Delta$ be a disk properly embedded in $C_{1}^{\prime} \cup_{f_{1}} H_{1}$ defined as follows.

- If $D_{a} \cap D_{1}=\emptyset$, let $\Delta=D_{a}$.

- If $D_{a} \cap D_{1} \neq \emptyset$, let $\Delta$ be the closure of a component of $D_{a} \backslash N\left(D_{1}\right)$ that is outermost in $D_{a}$.

Since $a \cap l_{1}=\emptyset$, the disk $\Delta$ is disjoint from $l_{1}$. Since $l_{0} \cup l_{1}$ is separating on $S$ by the condition (G2), and $a \neq l_{0}$, we see that $\Delta$ is essential in $C_{1}^{\prime} \cup_{f_{1}} H_{1}$.

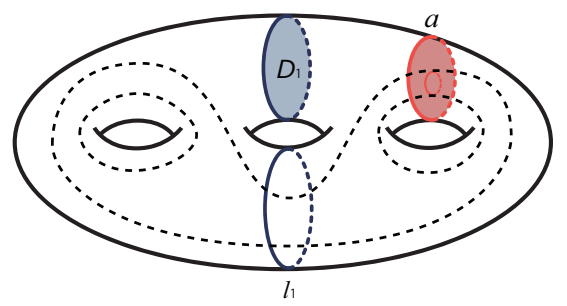

Fig. 1.

Since $C_{1}^{\prime}$ is homeomorphic to $\partial_{-} C_{1} \times[0,1]$, we may assume that $\Delta$ is obtained by gluing a vertical annulus in $C_{1}^{\prime}$ and an essential disk $\Delta^{\prime}$ in $H_{1}$ via $f_{1}$, after boundary compressions and isotopies toward $\partial_{-} C_{1}$ if necessary. This together with $\Delta \cap l_{1}=\emptyset$ implies that $d_{\partial_{-} C_{1}}\left(f_{1}\left(\partial \Delta^{\prime}\right), P_{1}\left(l_{1}\right)\right) \leq 1$. Since $f_{1}\left(\partial \Delta^{\prime}\right) \in f_{1}\left(\mathcal{D}\left(H_{1}\right)\right)$, we have $d_{\partial_{-} C_{1}}\left(f_{1}\left(\mathcal{D}\left(H_{1}\right)\right), P_{1}\left(l_{1}\right)\right) \leq 1$, a contradiction to the inequality (11). Q.E.D.

Let $\pi_{F_{1}}=\pi_{0} \circ \pi_{A}: \mathcal{C}^{0}(S) \rightarrow \mathcal{P}\left(\mathcal{A C}^{0}\left(F_{1}\right)\right) \rightarrow \mathcal{P}\left(\mathcal{C}^{0}\left(F_{1}\right)\right)$ be the subsurface projection introduced in Section 2. Recall that $\pi_{F_{1}}\left(l_{0}\right)=\left\{l_{0}\right\}$ since $l_{0} \cap l_{1}=\emptyset$.

Claim 4.2. For any element $a \in \mathcal{D}\left(V_{1}\right)$, we have $\pi_{F_{1}}(a) \neq \emptyset$, and $\operatorname{diam}_{F_{1}}\left(l_{0}, \pi_{F_{1}}(a)\right) \leq$ 4. 
Proof. Note that, by Claim 4.1 we immediately have $\pi_{F_{1}}(a) \neq \emptyset$. If $a=l_{0}$ or $a \cap l_{0}=\emptyset$, that is, $d_{S}\left(l_{0}, a\right) \leq 1$, then we have $\operatorname{diam}_{F_{1}}\left(l_{0}, \pi_{F_{1}}(a)\right) \leq 2$ by Lemma 2.2 . Hence, we suppose that $a \neq l_{0}$ and $a \cap l_{0} \neq \emptyset$ in the following.

Let $D_{a}$ be a disk in $V_{1}$ bounded by $a$, and recall that $l_{0}$ bounds the disk $D_{1}$ in $V_{1}$. Here, we may assume that $\left|a \cap l_{1}\right|=\left|a \cap N\left(l_{1}\right)\right|$ and is minimal. We may also assume that $\left|D_{a} \cap D_{1}\right|=\left|D_{a} \cap N\left(D_{1}\right)\right|$ and is minimal. Let $\Delta$ be the closure of a component of $D_{a} \backslash N\left(D_{1}\right)$ that is outermost in $D_{a}$. If $\Delta \cap l_{1}=\emptyset$, then we can lead to a contradiction by arguments in the proof of Claim 4.1 Hence, $\Delta \cap l_{1} \neq \emptyset$. Since $l_{0} \cup l_{1}$ is separating on $S$ by the condition (G2), there exists a component $\gamma$ of $\mathrm{Cl}\left(\partial \Delta \backslash\left(N\left(D_{1}\right) \cup N\left(l_{1}\right)\right)\right)$ such that $\partial \gamma \subset \partial N\left(l_{1}\right)$. It is clear that $\gamma$ is an essential arc on $F_{1}$. Note that $\gamma$ is disjoint from $l_{0}$, that is, $d_{\mathcal{A C}\left(F_{1}\right)}\left(l_{0}, \gamma\right)=1$, since $l_{0} \cap \Delta=\emptyset$ and $\gamma$ is a subarc of $\partial \Delta$. Since $\gamma \in \pi_{A}(a)$, we have $d_{\mathcal{A C}\left(F_{1}\right)}\left(l_{0}, \pi_{A}(a)\right) \leq$ $d_{\mathcal{A C}\left(F_{1}\right)}\left(l_{0}, \gamma\right)=1$. Hence,

$$
\operatorname{diam}_{\mathcal{A C}\left(F_{1}\right)}\left(l_{0}, \pi_{A}(a)\right) \leq d_{\mathcal{A C}\left(F_{1}\right)}\left(l_{0}, \pi_{A}(a)\right)+\operatorname{diam}_{\mathcal{A C}\left(F_{1}\right)}\left(\pi_{A}(a)\right) \leq 1+1=2 .
$$

By Lemma 2.1. we have $\operatorname{diam}_{F_{1}}\left(l_{0}, \pi_{F_{1}}(a)\right) \leq 4$.

Q.E.D.

Lemma 4.3. $d_{S}\left(\mathcal{D}\left(V_{1}\right), l_{n}\right)=n$.

Proof. Since $l_{0} \in \mathcal{D}\left(V_{1}\right)$, we have $d_{S}\left(\mathcal{D}\left(V_{1}\right), l_{n}\right) \leq n$. To prove $d_{S}\left(\mathcal{D}\left(V_{1}\right), l_{n}\right)=$ $n$, assume on the contrary that $d_{S}\left(\mathcal{D}\left(V_{1}\right), l_{n}\right)<n$. Then there exists a geodesic $\left[m_{0}, m_{1}, \ldots, m_{p}\right]$ in $\mathcal{C}(S)$ such that $p<n, m_{0} \in \mathcal{D}\left(V_{1}\right)$ and $m_{p}=l_{n}$.

Claim 4.4. $m_{i}=l_{1}$ for some $i \in\{0,1, \ldots, p\}$.

Proof. Assume on the contrary that $m_{i} \neq l_{1}$ for every $i \in\{0,1, \ldots, p\}$. Namely, every $m_{i}$ cuts $F_{1}$. By Lemma 2.2, we have

$$
\operatorname{diam}_{F_{1}}\left(\pi_{F_{1}}\left(m_{0}\right), \pi_{F_{1}}\left(m_{p}\right)\right) \leq 2 p .
$$

Similarly, we have

$$
\operatorname{diam}_{F_{1}}\left(\pi_{F_{1}}\left(l_{n}\right), \pi_{F_{1}}\left(l_{k}\right)\right) \leq 2(n-k) .
$$

By the triangle inequality, we have

$$
\begin{aligned}
\operatorname{diam}_{F_{1}}\left(\pi_{F_{1}}\left(l_{0}\right), \pi_{F_{1}}\left(l_{k}\right)\right) \leq & \operatorname{diam}_{F_{1}}\left(\pi_{F_{1}}\left(l_{0}\right), \pi_{F_{1}}\left(m_{0}\right)\right) \\
& +\operatorname{diam}_{F_{1}}\left(\pi_{F_{1}}\left(m_{0}\right), \pi_{F_{1}}\left(m_{p}\right)\right) \\
& +\operatorname{diam}_{F_{1}}\left(\pi_{F_{1}}\left(l_{n}\right), \pi_{F_{1}}\left(l_{k}\right)\right) .
\end{aligned}
$$

By the inequalities (2), (3), (4) and Claim 4.2 we obtain

$$
\begin{aligned}
\operatorname{diam}_{F_{1}}\left(\pi_{F_{1}}\left(l_{0}\right), \pi_{F_{1}}\left(l_{k}\right)\right) & \leq 4+2 p+2(n-k) \\
& <4+2 n+2 n,
\end{aligned}
$$

which contradicts the condition (G3).

Q.E.D.

By Claim 4.4 we have $d_{S}\left(m_{i}, m_{p}\right)=d_{S}\left(l_{1}, l_{n}\right)$. Since $\left[m_{0}, m_{1}, \ldots, m_{p}\right]$ and $\left[l_{0}, l_{1}, \ldots, l_{n}\right]$ are geodesics, $d_{S}\left(m_{i}, m_{p}\right)=p-i$ and $d_{S}\left(l_{1}, l_{n}\right)=n-1>p-1$. Hence, $p-i>p-1$, which implies $i=0$, that is, $m_{0}=l_{1}$. This contradicts Claim 4.1. Hence, we have $d_{S}\left(\mathcal{D}\left(V_{1}\right), l_{n}\right)=n$.

Q.E.D. 
Note that $f^{-1}\left(l_{n-1}\right)$ represents an essential simple closed curve on $X_{2}$. Since $f^{-1}\left(l_{n-1}\right)$ is non-separating on $\partial_{+} C_{2}$ by the condition (G1), $P_{2}\left(f^{-1}\left(l_{n-1}\right)\right)$ is an essential simple closed curve on $\partial_{-} C_{2}$. By [1], there exists a homeomorphism $f_{2}$ : $\partial H_{2} \rightarrow \partial_{-} C_{2}$ such that

$$
d_{\partial_{-} C_{2}}\left(f_{2}\left(\mathcal{D}\left(H_{2}\right)\right), P_{2}\left(f^{-1}\left(l_{n-1}\right)\right)\right) \geq 2 .
$$

Let $V_{2}=C_{2} \cup_{f_{2}} H_{2}$. Then $V_{1} \cup_{f} V_{2}$ is a genus- $g$ Heegaard splitting.

Claims 4.5, 4.6 and Lemma 4.7 below can be proved by the arguments similar to those for Claims 4.1, 4.2 and Lemma 4.3, respectively.

Claim 4.5. $l_{n-1}$ intersects every element of $f\left(\mathcal{D}\left(V_{2}\right)\right) \backslash\left\{l_{n}\right\}$.

Claim 4.6. For any element $a \in f\left(\mathcal{D}\left(V_{2}\right)\right)$, we have $\pi_{F_{n-1}}(a) \neq \emptyset$, and $\operatorname{diam}_{F_{n-1}}\left(l_{n}, \pi_{F_{n-1}}(a)\right) \leq 4$.

Lemma 4.7. $d_{S}\left(f\left(\mathcal{D}\left(V_{2}\right)\right), l_{0}\right)=n$.

Claim 4.8. $\operatorname{diam}_{F_{1}}\left(\pi_{F_{1}}\left(f\left(\mathcal{D}\left(V_{2}\right)\right)\right)\right) \leq 12$ and $\operatorname{diam}_{F_{n-1}}\left(\pi_{F_{n-1}}\left(\mathcal{D}\left(V_{1}\right)\right)\right) \leq 12$.

Proof. By Lemma 4.3, we have $d_{S}\left(\mathcal{D}\left(V_{1}\right), l_{n-1}\right)=n-1 \geq 3$. Hence, by 8 , Theorem 1], $\operatorname{diam}_{F_{n-1}}\left(\pi_{F_{n-1}}\left(\mathcal{D}\left(V_{1}\right)\right)\right) \leq 12$. Similarly, we have $\operatorname{diam}_{F_{1}}\left(\pi_{F_{1}}\left(f\left(\mathcal{D}\left(V_{2}\right)\right)\right) \leq\right.$ 12 by Lemma 4.7 and 8 .

Q.E.D.

Lemma 4.9. $d_{S}\left(\mathcal{D}\left(V_{1}\right), f\left(\mathcal{D}\left(V_{2}\right)\right)\right)=n$. Namely, the Hempel distance of the Heegaard splitting $V_{1} \cup_{f} V_{2}$ is $n$.

Proof. Since $l_{0} \in \mathcal{D}\left(V_{1}\right)$ and $l_{n} \in f\left(\mathcal{D}\left(V_{2}\right)\right)$, we have $d_{S}\left(\mathcal{D}\left(V_{1}\right), f\left(\mathcal{D}\left(V_{2}\right)\right)\right) \leq n$. Let $\left[m_{0}, m_{1}, \ldots, m_{p}\right]$ be a geodesic in $\mathcal{C}(S)$ such that $m_{0} \in \mathcal{D}\left(V_{1}\right), m_{p} \in f\left(\mathcal{D}\left(V_{2}\right)\right)$ and $p \leq n$.

Claim 4.10. $m_{i}=l_{1}$ for some $i \in\{0,1, \ldots, p\}$.

Proof. Assume on the contrary that $m_{i} \neq l_{1}$ for every $i \in\{0,1, \ldots, p\}$. Namely, every $m_{i}$ cuts $F_{1}$. By Lemma 2.2, we have

$$
\operatorname{diam}_{F_{1}}\left(\pi_{F_{1}}\left(m_{0}\right), \pi_{F_{1}}\left(m_{p}\right)\right) \leq 2 p .
$$

Recall that $k \in\{2,3, \ldots, n-2\}$. Similarly, we have

$$
\operatorname{diam}_{F_{1}}\left(\pi_{F_{1}}\left(l_{n}\right), \pi_{F_{1}}\left(l_{k}\right)\right) \leq 2(n-k) .
$$

By the triangle inequality, we have

$$
\begin{aligned}
\operatorname{diam}_{F_{1}}\left(\pi_{F_{1}}\left(l_{0}\right), \pi_{F_{1}}\left(l_{k}\right)\right) \leq & \operatorname{diam}_{F_{1}}\left(\pi_{F_{1}}\left(l_{0}\right), \pi_{F_{1}}\left(m_{0}\right)\right) \\
& +\operatorname{diam}_{F_{1}}\left(\pi_{F_{1}}\left(m_{0}\right), \pi_{F_{1}}\left(m_{p}\right)\right) \\
& +\operatorname{diam}_{F_{1}}\left(\pi_{F_{1}}\left(m_{p}\right), \pi_{F_{1}}\left(l_{n}\right)\right) \\
& +\operatorname{diam}_{F_{1}}\left(\pi_{F_{1}}\left(l_{n}\right), \pi_{F_{1}}\left(l_{k}\right)\right) .
\end{aligned}
$$

By the inequalities (7), (8), (9) together with Claims 4.2 and 4.8, we obtain

$$
\begin{aligned}
\operatorname{diam}_{F_{1}}\left(\pi_{F_{1}}\left(l_{0}\right), \pi_{F_{1}}\left(l_{k}\right)\right) & \leq 4+2 p+12+2(n-k) \\
& <4+2 n+12+2 n,
\end{aligned}
$$

which contradicts the condition (G3).

Q.E.D.

The following claim can be proved similarly. 
Claim 4.11. $m_{j}=l_{n-1}$ for some $j \in\{0,1, \ldots, p\}$.

Note that $l_{1} \notin \mathcal{D}\left(V_{1}\right)$ by Claim 4.1. Note also that $l_{1} \notin f\left(\mathcal{D}\left(V_{2}\right)\right)$ since, otherwise, we have $d_{S}\left(f\left(\mathcal{D}\left(V_{2}\right)\right), l_{0}\right) \leq d_{S}\left(l_{1}, l_{0}\right)=1$, which contradicts Lemma 4.7 Since $m_{0} \in \mathcal{D}\left(V_{1}\right)$ and $m_{p} \in f\left(\mathcal{D}\left(V_{2}\right)\right)$ by the assumption, we have $m_{i}\left(=l_{1}\right) \neq m_{0}$ and $m_{i}\left(=l_{1}\right) \neq m_{p}$, which implies $1 \leq i \leq p-1$. Similarly, we have $1 \leq j \leq p-1$. Hence, we have

$$
|i-j| \leq(p-1)-1=p-2 .
$$

On the other hand, by Claims 4.10 and 4.11 we have

$$
|i-j|=d_{S}\left(m_{i}, m_{j}\right)=d_{S}\left(l_{1}, l_{n-1}\right)=n-2,
$$

which together with the inequality (11) implies $p=n$. Hence, $d_{S}\left(\mathcal{D}\left(V_{1}\right), f\left(\mathcal{D}\left(V_{2}\right)\right)\right)=$ $n$.

Lemma 4.12. The Heegaard splitting $V_{1} \cup_{f} V_{2}$ is keen.

Proof. Let $\left[m_{0}, m_{1}, \ldots, m_{n}\right]$ be a geodesic in $\mathcal{C}(S)$ such that $m_{0} \in \mathcal{D}\left(V_{1}\right)$ and $m_{n} \in f\left(\mathcal{D}\left(V_{2}\right)\right)$. By the proof of Lemma 4.9 we have $m_{1}=l_{1}$ and $m_{n-1}=l_{n-1}$. By Claims 4.1 and 4.5. we have $m_{0}=l_{0}$ and $m_{n}=l_{n}$.

Q.E.D.

In the remainder of this section, we show that the existence of strongly keen Heegaard splitting.

Claim 4.13. In the above construction, if the following conditions are satisfied, then the Heegaard splitting constructed from the geodesic $\left[l_{0}, l_{1}, \ldots, l_{n}\right]$ is strongly keen.

- The geodesic $\left[l_{1}^{\prime}, l_{2}^{\prime}, \ldots, l_{k}^{\prime}\right]$ (resp. $\left[l_{1}^{\prime \prime}, l_{2}^{\prime \prime}, \ldots, l_{n-k}^{\prime \prime}\right]$ ) is the unique geodesic from $l_{0}^{\prime}$ to $l_{k}^{\prime}$ (resp. $l_{0}^{\prime \prime}$ to $l_{n-k}^{\prime \prime}$ ).

Proof. By the proof of Lemma 4.12. $m_{i}=l_{i}$ holds for $i=0,1, n-1$ and $n$. Moreover, by the condition (G5) and Remark 2.5, we have $m_{k}=l_{k}$. Hence, if the geodesics $\left[l_{1}^{\prime}, l_{2}^{\prime}, \ldots, l_{k}^{\prime}\right]$ (resp. $\left.\left[l_{1}^{\prime \prime}, l_{2}^{\prime \prime}, \ldots, l_{n-k}^{\prime \prime}\right]\right)$ is the unique geodesic containing $l_{0}^{\prime}$ and $l_{k}^{\prime}$ (resp. $l_{0}^{\prime \prime}$ and $l_{n-k}^{\prime \prime}$ ), then we obtain the desired result.

Q.E.D.

Hence the next claim completes the proof of Theorem 1.1

Claim 4.14. For each $p$, there exists a geodesic $\left[\alpha_{0}, \alpha_{1}, \ldots, \alpha_{p}\right]$ such that each $\alpha_{i}(i=0,1, \ldots, p)$ is non-separating on $S$ and $\left[\alpha_{0}, \alpha_{1}, \ldots, \alpha_{p}\right]$ is the unique geodesic connecting $\alpha_{0}$ and $\alpha_{p}$.

Proof. Let $\alpha_{0}$ and $\alpha_{1}$ be non-separating simple closed curve on $S$ such that $\alpha_{0} \cap \alpha_{1}=\emptyset$, and let $X_{1}=\operatorname{Cl}\left(S \backslash N\left(\alpha_{1}\right)\right)$. Let $\alpha_{2}^{\prime}$ be a non-separating simple closed curve on $S$ disjoint from $\alpha_{1}$. By [9, Proposition 4.6], there exists a homeomorphism $g_{1}: S \rightarrow S$ such that $g_{1}\left(\alpha_{1}\right)=\alpha_{1}$ and $\operatorname{diam}_{X_{1}}\left(\pi_{X_{1}}\left(\alpha_{0}\right), \pi_{X_{1}}\left(g_{1}\left(\alpha_{2}^{\prime}\right)\right)\right)>4$. Let $\alpha_{2}=g_{1}\left(\alpha_{2}^{\prime}\right)$. By Lemma 2.3. $\left[\alpha_{0}, \alpha_{1}, \alpha_{2}\right]$ is a geodesic in $\mathcal{C}(S)$. Moreover, by Remark 2.5, $\left[\alpha_{0}, \alpha_{1}, \alpha_{2}\right]$ is the unique geodesic connecting $\alpha_{0}$ and $\alpha_{2}$.

For any positive integer $p$, we repeat this process to construct a geodesic $\left[\alpha_{0}, \alpha_{1}, \ldots, \alpha_{p}\right]$ inductively as follows. Suppose we have constructed a geodesic $\left[\alpha_{0}, \alpha_{1}, \ldots, \alpha_{i}\right]$ for $i<p$ such that

- $\alpha_{i}$ is non-separating on $S$, and

- $\left[\alpha_{0}, \alpha_{1}, \ldots, \alpha_{i}\right]$ is the unique geodesic connecting $\alpha_{0}$ and $\alpha_{i}$. 
Let $X_{i}=\mathrm{Cl}\left(S \backslash N\left(\alpha_{i}\right)\right)$. Let $\alpha_{i+1}^{\prime}$ be a non-separating simple closed curve on $S$ disjoint from $\alpha_{i}$. By [9, Proposition 4.6], there exists a homeomorphism $g_{i}$ : $S \rightarrow S$ such that $g_{i}\left(\alpha_{i}\right)=\alpha_{i}$ and $\operatorname{diam}_{X_{i}}\left(\pi_{X_{i}}\left(\alpha_{0}\right), \pi_{X_{i}}\left(g_{i}\left(\alpha_{i+1}^{\prime}\right)\right)\right)>2(i+1)$. Let $\alpha_{i+1}=g_{i}\left(\alpha_{i+1}^{\prime}\right)$. By Lemma 2.3. $\left[\alpha_{0}, \alpha_{1}, \ldots, \alpha_{i+1}\right]$ is a geodesic in $\mathcal{C}(S)$. Moreover, by Remark 2.5, every geodesic connecting $\alpha_{0}$ and $\alpha_{i+1}$ passes through $\alpha_{i}$. Since $\left[\alpha_{0}, \alpha_{1}, \ldots, \alpha_{i}\right]$ is the unique geodesic connecting $\alpha_{0}$ and $\alpha_{i}$, we have that $\left[\alpha_{0}, \alpha_{1}, \ldots, \alpha_{i+1}\right]$ is the unique geodesic connecting $\alpha_{0}$ and $\alpha_{i+1}$. Hence, we obtain a geodesic $\left[\alpha_{0}, \alpha_{1}, \ldots, \alpha_{p}\right]$ such that every $\alpha_{i}(i=0,1, \ldots, p)$ is non-separating on $S$ and $\left[\alpha_{0}, \alpha_{1}, \ldots, \alpha_{p}\right]$ is the unique geodesic connecting $\alpha_{0}$ and $\alpha_{p}$.

Q.E.D.

\section{$\S 5 . \quad$ Proof of Theorem 1.1 when $n=2$}

Let $n=2$ and $g$ be an integer with $g \geq 3$. Let $S$ be a closed connected orientable surface of genus $g$. Let $l_{0}$ and $l_{1}$ be non-separating simple closed curves on $S$ such that $l_{0} \cup l_{1}$ is separating on $S$ and $l_{0}, l_{1}$ are not parallel on $S$. By 9 , Proposition 4.6], there exists a homeomorphism $h: S \rightarrow S$ such that $h\left(l_{1}\right)=l_{1}$ and

$$
d_{F_{1}}\left(l_{0}, h\left(l_{0}\right)\right)>12
$$

where $F_{1}=\mathrm{Cl}\left(S \backslash N\left(l_{1}\right)\right)$. Let $l_{2}=h\left(l_{0}\right)$. By Lemma 2.3, $\left[l_{0}, l_{1}, l_{2}\right]$ is a geodesic in $\mathcal{C}(S)$.

Let $C_{1}$ and $C_{2}$ be copies of the compression-body obtained by adding a 1-handle to $F \times[0,1]$, where $F$ is a closed orientable surface of genus $g-1$. Let $D_{1}$ and $D_{2}$ be the non-separating essential disk properly embedded in $C_{1}$ and $C_{2}$ corresponding to the co-cores of the 1-handles, respectively. We may assume that $\partial_{+} C_{1}=S$ and $\partial D_{1}=l_{0}$. Choose a homeomorphism $f: \partial_{+} C_{2} \rightarrow \partial_{+} C_{1}$ such that $f\left(\partial D_{2}\right)=l_{2}$.

Let $H_{i}, C_{i}^{\prime}, X_{i}, P_{i}(i=1,2)$ be as in Section 4 . Note that $l_{1}$ is non-separating on $S$, and hence, $P_{1}\left(l_{1}\right)$ and $P_{2}\left(f^{-1}\left(l_{1}\right)\right)$ are essential simple closed curves on $\partial_{-} C_{1}$ and $\partial_{-} C_{2}$, respectively. By [1], there exist homeomorphisms $f_{1}: \partial H_{1} \rightarrow \partial_{-} C_{1}$ and $f_{2}$ : $\partial H_{2} \rightarrow \partial_{-} C_{2}$ such that $d_{\partial_{-} C_{1}}\left(f_{1}\left(\mathcal{D}\left(H_{1}\right)\right), P_{1}\left(l_{1}\right)\right) \geq 2$ and $d_{\partial_{-} C_{2}}\left(f_{2}\left(\mathcal{D}\left(H_{2}\right)\right), P_{2}\left(f^{-1}\left(l_{1}\right)\right)\right) \geq$ 2 , respectively. Let $V_{i}=C_{i} \cup_{f_{i}} H_{i}(i=1,2)$. Then, $V_{1} \cup_{f} V_{2}$ is a genus- $g$ Heegaard splitting. By the arguments similar to those for Claims 4.1, 4.2, 4.5] and 4.6, we obtain the following.

Claim 5.1. (1) $l_{1}$ intersects every element of $\mathcal{D}\left(V_{1}\right) \backslash\left\{l_{0}\right\}$ and every element of $f\left(\mathcal{D}\left(V_{2}\right)\right) \backslash\left\{l_{2}\right\}$.

(2) For any element $a \in \mathcal{D}\left(V_{1}\right)$, we have $\pi_{F_{1}}(a) \neq \emptyset$, and $\operatorname{diam}_{F_{1}}\left(l_{0}, \pi_{F_{1}}(a)\right) \leq$ 4.

(3) For any element $a \in f\left(\mathcal{D}\left(V_{2}\right)\right)$, we have $\pi_{F_{1}}(a) \neq \emptyset$, and $\operatorname{diam}_{F_{1}}\left(l_{2}, \pi_{F_{1}}(a)\right) \leq$ 4.

Lemma 5.2. $V_{1} \cup_{f} V_{2}$ is a strongly keen Heegaard splitting whose Hempel distance is 2 .

Proof. Since $l_{0} \in \mathcal{D}\left(V_{1}\right)$ and $l_{2} \in f\left(\mathcal{D}\left(V_{2}\right)\right)$, we have $d_{S}\left(\mathcal{D}\left(V_{1}\right), f\left(\mathcal{D}\left(V_{2}\right)\right)\right) \leq 2$. Let $\left[m_{0}, m_{1}, m_{2}\right]$ be a geodesic in $\mathcal{C}(S)$ such that $m_{0} \in \mathcal{D}\left(V_{1}\right)$ and $m_{2} \in f\left(\mathcal{D}\left(V_{2}\right)\right)$. (Possibly, $m_{1} \in \mathcal{D}\left(V_{1}\right)$ or $m_{1} \in f\left(\mathcal{D}\left(V_{2}\right)\right)$.) By Claim 5.1 (1), both $m_{0}$ and $m_{2}$ cut $F_{1}$. If $m_{1}$ also cuts $F_{1}$, then we have $\operatorname{diam}_{F_{1}}\left(\pi_{F_{1}}\left(m_{0}\right), \pi_{F_{1}}\left(m_{2}\right)\right) \leq 4$ by Lemma 2.2 
which together with Claim 5.1 (2) and (3) implies that

$$
\begin{aligned}
d_{F_{1}}\left(l_{0}, l_{2}\right) \leq & \operatorname{diam}_{F_{1}}\left(l_{0}, \pi_{F_{1}}\left(m_{0}\right)\right)+\operatorname{diam}_{F_{1}}\left(\pi_{F_{1}}\left(m_{0}\right), \pi_{F_{1}}\left(m_{2}\right)\right) \\
& +\operatorname{diam}_{F_{1}}\left(\pi_{F_{1}}\left(m_{2}\right), l_{2}\right) \\
\leq & 4+4+4=12 .
\end{aligned}
$$

This contradicts the fact that $d_{F_{1}}\left(l_{0}, l_{2}\right)>12$. Hence, $m_{1}$ misses $F_{1}$, that is, $m_{1}=l_{1}$. By Claim 5.1 (1), we have $m_{0}=l_{0}$ and $m_{2}=l_{2}$, and we obtain the desired result.

Q.E.D.

\section{§6. Proof of Theorem 1.1 when $n=3$}

Let $n=3$ and $g$ be an integer with $g \geq 3$. Let $S$ be a closed connected orientable surface of genus $g$. Let $l_{0}$ and $l_{1}$ be non-separating simple closed curves on $S$ such that $l_{0} \cup l_{1}$ is separating on $S$ and $l_{0}, l_{1}$ are not parallel on $S$. Let $l_{2}^{\prime}$ be a simple closed curve on $S$ such that $l_{2}^{\prime} \cap l_{1}=\emptyset$ and $l_{1} \cup l_{2}^{\prime}$ is non-separating on $S$. By [9, Proposition 4.6], there exists a homeomorphism $h_{1}: S \rightarrow S$ such that $h_{1}\left(l_{1}\right)=l_{1}$ and

$$
d_{F_{1}}\left(l_{0}, h_{1}\left(l_{2}^{\prime}\right)\right)>8
$$

where $F_{1}=\mathrm{Cl}\left(S \backslash N\left(l_{1}\right)\right)$. Let $l_{2}=h_{1}\left(l_{2}^{\prime}\right)$. By Lemma 2.3. $\left[l_{0}, l_{1}, l_{2}\right]$ is a geodesic in $\mathcal{C}(S)$. Note that there exists a homeomorphism $h_{2}: S \rightarrow S$ such that $h_{2}\left(l_{1}\right)=l_{2}$ and $h_{2}\left(l_{2}\right)=l_{1}$, since $l_{1} \cup l_{2}$ is non-separating on $S$. Let $l_{3}^{\prime}=h_{2}\left(l_{0}\right)$. Note that $\left[l_{1}, l_{2}, l_{3}^{\prime}\right]$ is a geodesic in $\mathcal{C}(S)$.

Let $S^{\prime}=\mathrm{Cl}\left(S \backslash N\left(l_{1} \cup l_{2}\right)\right)$. Let $\pi_{S^{\prime}}=\pi_{0} \circ \pi_{A}: \mathcal{C}^{0}(S) \rightarrow \mathcal{P}\left(\mathcal{A C}^{0}\left(S^{\prime}\right)\right) \rightarrow$ $\mathcal{P}\left(\mathcal{C}^{0}\left(S^{\prime}\right)\right)$ be the subsurface projection introduced in Section 2 ,

Claim 6.1. There exists a homeomorphism $h: S \rightarrow S$ such that $h\left(l_{1}\right)=l_{1}$, $h\left(l_{2}\right)=l_{2}$ and $\operatorname{diam}_{S^{\prime}}\left(\pi_{S^{\prime}}\left(l_{0}\right), \pi_{S^{\prime}}\left(h\left(l_{3}^{\prime}\right)\right)\right)>14$.

Proof. Let $\gamma$ be the closure of a component of $l_{3}^{\prime} \backslash l_{1}$. Since $l_{3}^{\prime} \cap l_{2}=\emptyset$, we have $\gamma \in \pi_{A}\left(l_{3}^{\prime}\right)$, and hence, $\pi_{0}(\gamma) \in \pi_{0}\left(\pi_{A}\left(l_{3}^{\prime}\right)\right)=\pi_{S^{\prime}}\left(l_{3}^{\prime}\right)$. Note that $\pi_{0}(\gamma)$ consists of a single simple closed curve or two disjoint simple closed curves on $S^{\prime}$. Since the diameter of $\mathcal{C}\left(S^{\prime}\right)$ is infinite, there exists a homeomorphism $h: S \rightarrow S$ such that $h\left(l_{1}\right)=l_{1}, h\left(l_{2}\right)=l_{2}$ and $d_{S^{\prime}}\left(\pi_{S^{\prime}}\left(l_{0}\right), h\left(\pi_{0}(\gamma)\right)\right)>14$. This inequality, together with the fact that $h\left(\pi_{0}(\gamma)\right) \in h\left(\pi_{S^{\prime}}\left(l_{3}^{\prime}\right)\right)$, implies

$$
\begin{aligned}
\operatorname{diam}_{S^{\prime}}\left(\pi_{S^{\prime}}\left(l_{0}\right), \pi_{S^{\prime}}\left(h\left(l_{3}^{\prime}\right)\right)\right) & =\operatorname{diam}_{S^{\prime}}\left(\pi_{S^{\prime}}\left(l_{0}\right), h\left(\pi_{S^{\prime}}\left(l_{3}^{\prime}\right)\right)\right) \\
& \geq d_{S^{\prime}}\left(\pi_{S^{\prime}}\left(l_{0}\right), h\left(\pi_{0}(\gamma)\right)\right) \\
& >14 .
\end{aligned}
$$

Q.E.D.

Let $l_{3}=h\left(l_{3}^{\prime}\right)$. By Lemma 2.4 $\left[l_{0}, l_{1}, l_{2}, l_{3}\right]$ is a geodesic in $\mathcal{C}(S)$. Note that the following hold.

- $d_{F_{1}}\left(l_{0}, l_{2}\right)>8$.

- $d_{F_{2}}\left(l_{1}, l_{3}\right)>8$, where $F_{2}=\mathrm{Cl}\left(S \backslash N\left(l_{2}\right)\right)$, since $d_{F_{1}}\left(l_{0}, l_{2}\right)>8$ and the homeomorphism $h \circ h_{2}$ sends $l_{0}, l_{1}, l_{2}$ to $l_{3}, l_{2}, l_{1}$, respectively.

- $\operatorname{diam}_{S^{\prime}}\left(\pi_{S^{\prime}}\left(l_{0}\right), \pi_{S^{\prime}}\left(l_{3}\right)\right)>14$.

Let $C_{1}$ and $C_{2}$ be copies of the compression-body obtained by adding a 1-handle to $F \times[0,1]$, where $F$ is a closed orientable surface of genus $g-1$. Let $D_{1}$ and $D_{2}$ be 
the non-separating essential disk properly embedded in $C_{1}$ and $C_{2}$ corresponding to the co-cores of the 1-handles, respectively. We may assume that $\partial_{+} C_{1}=S$ and $\partial D_{1}=l_{0}$. Choose a homeomorphism $f: \partial_{+} C_{2} \rightarrow \partial_{+} C_{1}$ such that $f\left(\partial D_{2}\right)=l_{3}$.

Let $H_{i}, C_{i}^{\prime}, X_{i}, P_{i}(i=1,2)$ be as in Section 4 . Note that $l_{1}$ and $l_{2}$ are nonseparating on $S$ and not isotopic to $l_{0}$ or $l_{3}$. Hence, $P_{1}\left(l_{1}\right)$ and $P_{2}\left(f^{-1}\left(l_{2}\right)\right)$ are essential simple closed curves on $\partial_{-} C_{1}$ and $\partial_{-} C_{2}$, respectively. By 1 , there exist homeomorphisms $f_{1}: \partial H_{1} \rightarrow \partial_{-} C_{1}$ and $f_{2}: \partial H_{2} \rightarrow \partial_{-} C_{2}$ such that $d_{\partial_{-} C_{1}}\left(f_{1}\left(\mathcal{D}\left(H_{1}\right)\right), P_{1}\left(l_{1}\right)\right) \geq 2$ and $d_{\partial_{-} C_{2}}\left(f_{2}\left(\mathcal{D}\left(H_{2}\right)\right), P_{2}\left(f^{-1}\left(l_{2}\right)\right)\right) \geq 2$, respectively. Let $V_{i}=C_{i} \cup_{f_{i}} H_{i}(i=1,2)$. Then, $V_{1} \cup_{f} V_{2}$ is a genus- $g$ Heegaard splitting. By the arguments similar to those for Claims 4.1 4.2, 4.5 and 4.6. we obtain the following.

Claim 6.2. (1) $l_{1}$ intersects every element of $\mathcal{D}\left(V_{1}\right) \backslash\left\{l_{0}\right\}$, and $l_{2}$ intersects every element of $f\left(\mathcal{D}\left(V_{2}\right)\right) \backslash\left\{l_{3}\right\}$.

(2) For any element $a \in \mathcal{D}\left(V_{1}\right)$, we have $\pi_{F_{1}}(a) \neq \emptyset$, and $\operatorname{diam}_{F_{1}}\left(l_{0}, \pi_{F_{1}}(a)\right) \leq$ 4 .

(3) For any element $a \in f\left(\mathcal{D}\left(V_{2}\right)\right)$, we have $\pi_{F_{2}}(a) \neq \emptyset$, and $\operatorname{diam}_{F_{2}}\left(l_{3}, \pi_{F_{2}}(a)\right) \leq$

Lemma 6.3. (1) For any element $a \in \mathcal{D}\left(V_{1}\right)$, we have $\pi_{S^{\prime}}\left(l_{0}\right) \neq \emptyset, \pi_{S^{\prime}}(a) \neq \emptyset$, and $\operatorname{diam}_{S^{\prime}}\left(\pi_{S^{\prime}}\left(l_{0}\right), \pi_{S^{\prime}}(a)\right) \leq 4$.

(2) For any element $a \in f\left(\mathcal{D}\left(V_{2}\right)\right)$, we have $\pi_{S^{\prime}}\left(l_{2}\right) \neq \emptyset, \pi_{S^{\prime}}(a) \neq \emptyset$, and $\operatorname{diam}_{S^{\prime}}\left(\pi_{S^{\prime}}\left(l_{2}\right), \pi_{S^{\prime}}(a)\right) \leq 4$.

Proof. We give a proof for (1) only, since (2) can be proved similarly. Suppose that $\pi_{S^{\prime}}\left(l_{0}\right)=\emptyset\left(\right.$ resp. $\left.\pi_{S^{\prime}}(a)=\emptyset\right)$. This means that for each component $\gamma$ of $l_{0} \cap S^{\prime}$ (resp. $a \cap S^{\prime}$ ), each component of $S^{\prime} \backslash \gamma$ is an annulus. This shows that $S^{\prime}$ is a sphere with three boundary components, a contradiction. If $a=l_{0}$ or $a \cap l_{0}=\emptyset$, then we have $\operatorname{diam}_{S^{\prime}}\left(\pi_{S^{\prime}}\left(l_{0}\right), \pi_{S^{\prime}}(a)\right) \leq 2$ by Lemma 2.2. Hence, we suppose that $a \neq l_{0}$ and $a \cap l_{0} \neq \emptyset$ in the following.

Let $D_{a}$ be a disk in $V_{1}$ bounded by $a$, and recall $l_{0}$ bounds the disk $D_{1}$ in $V_{1}$. We may assume that $\left|D_{a} \cap D_{1}\right|$ is minimal. Let $\Delta$ be the closure of a component of $D_{a} \backslash D_{1}$ that is outermost in $D_{a}$. Let $D_{1}^{(1)}$ and $D_{1}^{(2)}$ be the components of $D_{1} \backslash \Delta$. By the minimality of $\left|D_{a} \cap D_{1}\right|$, the disks $D_{1}^{(1)} \cup \Delta$ and $D_{1}^{(2)} \cup \Delta$ are essential in $V_{1}$

Claim 6.4. $D_{1}^{(1)} \cup \Delta$ or $D_{1}^{(2)} \cup \Delta$, say $D_{1}^{(1)} \cup \Delta$, is not isotopic to $D_{1}$ in $V_{1}$.

Proof. Let $m_{1}$ and $m_{2}$ be the two simple closed curves obtained from $l_{0}$ (= $\left.\partial D_{1}\right)$ by a band move along $\Delta \cap \partial V_{1}$. Suppose both $D_{1}^{(1)} \cup \Delta$ and $D_{1}^{(2)} \cup \Delta$ are isotopic to $D_{1}$ in $V_{1}$. This implies that $m_{1}$ and $m_{2}$ are parallel in $\partial V_{1}$, and hence, they co-bound an annulus, say $A$, in $S$. Further, by slight isotopy, we may suppose that $l_{0} \cap\left(m_{1} \cup m_{2}\right)=\emptyset$. Note that $l_{0}$ is retrieved from $m_{1} \cup m_{2}$ by a band move along an arc $\alpha$ such that $\left|\alpha \cap\left(\Delta \cap \partial V_{1}\right)\right|=1$. Since $l_{0}$ is essential, (int $\left.\alpha\right) \cap A=\emptyset$. This shows that $l_{0}$ cuts off a punctured torus from $\partial V_{1}$, which contradicts the assumption that $l_{0}$ is non-separating on $\partial V_{1}$.

Q.E.D.

Hence, by Claim 6.2 (1), $l_{1}$ intersects $D_{1}^{(1)} \cup \Delta$. Since $l_{1} \cap D_{1}=\emptyset, l_{1}$ intersects $\partial \Delta \backslash D_{1}$. Since $l_{0} \cup l_{1}$ is separating on $S$, there is a subarc $\gamma$ of $\partial \Delta \backslash D_{1}$ such that $\partial \gamma \subset l_{1}$. Let $\gamma^{\prime}$ be the closure of a component of $\gamma \backslash N\left(l_{1} \cup l_{2}\right)$. Then $\gamma^{\prime}$ is an element of $\pi_{A}(a)\left(\subset \mathcal{A C}^{0}\left(S^{\prime}\right)\right)$. Hence, we have

$$
\operatorname{diam}_{\mathcal{A C}\left(S^{\prime}\right)}\left(\gamma^{\prime}, \pi_{A}(a)\right) \leq 1 .
$$


On the other hand, since $\gamma^{\prime}$ is disjoint from $l_{0}$, we have

$$
\operatorname{diam}_{\mathcal{A C}\left(S^{\prime}\right)}\left(\pi_{A}\left(l_{0}\right), \gamma^{\prime}\right) \leq 1
$$

By the triangle inequality, we have

$$
\begin{aligned}
\operatorname{diam}_{\mathcal{A C}\left(S^{\prime}\right)}\left(\pi_{A}\left(l_{0}\right), \pi_{A}(a)\right) & \leq \operatorname{diam}_{\mathcal{A C}\left(S^{\prime}\right)}\left(\pi_{A}\left(l_{0}\right), \gamma^{\prime}\right)+\operatorname{diam}_{\mathcal{A C}\left(S^{\prime}\right)}\left(\gamma^{\prime}, \pi_{A}(a)\right) \\
& \leq 1+1=2 .
\end{aligned}
$$

By Lemma 2.1, we have $\operatorname{diam}_{S^{\prime}}\left(\pi_{S^{\prime}}\left(l_{0}\right), \pi_{S^{\prime}}(a)\right) \leq 4$. This completes the proof of Lemma $6.3(1)$.

Q.E.D.

Lemma 6.5. $V_{1} \cup_{f} V_{2}$ is a strongly keen Heegaard splitting whose Hempel distance is 3 .

Proof. Since $l_{0} \in \mathcal{D}\left(V_{1}\right)$ and $l_{3} \in f\left(\mathcal{D}\left(V_{2}\right)\right)$, we have $d_{S}\left(\mathcal{D}\left(V_{1}\right), f\left(\mathcal{D}\left(V_{2}\right)\right)\right) \leq 3$. Let $\left[m_{0}, \ldots, m_{p}\right]$ be a geodesic in $\mathcal{C}(S)$ such that $m_{0} \in \mathcal{D}\left(V_{1}\right), m_{p} \in f\left(\mathcal{D}\left(V_{2}\right)\right)$ and $p \leq 3$.

Claim 6.6. $m_{i}=l_{1}$ or $m_{i}=l_{2}$ for some $i \in\{0, \ldots, p\}$.

Proof. Assume on the contrary that $m_{i} \neq l_{1}$ and $m_{i} \neq l_{2}$ for every $i \in$ $\{0, \ldots, p\}$. Namely, every $m_{i}$ cuts $S^{\prime}$. By Lemma 2.2 we have

$$
\operatorname{diam}_{S^{\prime}}\left(\pi_{S^{\prime}}\left(m_{0}\right), \pi_{S^{\prime}}\left(m_{p}\right)\right) \leq 2 p \leq 6 .
$$

By the triangle inequality, we have

$$
\begin{aligned}
\operatorname{diam}_{S^{\prime}}\left(\pi_{S^{\prime}}\left(l_{0}\right), \pi_{S^{\prime}}\left(l_{3}\right)\right) \leq & \operatorname{diam}_{S^{\prime}}\left(\pi_{S^{\prime}}\left(l_{0}\right), \pi_{S^{\prime}}\left(m_{0}\right)\right) \\
& +\operatorname{diam}_{S^{\prime}}\left(\pi_{S^{\prime}}\left(m_{0}\right), \pi_{S^{\prime}}\left(m_{p}\right)\right) \\
& +\operatorname{diam}_{S^{\prime}}\left(\pi_{S^{\prime}}\left(m_{p}\right), \pi_{S^{\prime}}\left(l_{3}\right)\right) .
\end{aligned}
$$

By the inequalities (12), (13) together with Lemma 6.3, we obtain

$$
\operatorname{diam}_{S^{\prime}}\left(\pi_{S^{\prime}}\left(l_{0}\right), \pi_{S^{\prime}}\left(l_{3}\right)\right) \leq 4+6+4=14
$$

which contradicts the inequality $\operatorname{diam}_{S^{\prime}}\left(\pi_{S^{\prime}}\left(l_{0}\right), \pi_{S^{\prime}}\left(l_{3}\right)\right)>14$ (see Claim 6.1).

Q.E.D.

Assume that $m_{i}=l_{1}$ for some $i \in\{0, \ldots, p\}$. (The case where $m_{i}=l_{2}$ for some $i \in\{0, \ldots, p\}$ can be treated similarly.) Since $m_{0} \in \mathcal{D}\left(V_{1}\right)$, we have

$$
d_{S}\left(\mathcal{D}\left(V_{1}\right), m_{i}\right) \leq d_{S}\left(m_{0}, m_{i}\right)=i .
$$

On the other hand, by Claim 6.6 and Claim 6.2 (1), we have

$$
d_{S}\left(\mathcal{D}\left(V_{1}\right), m_{i}\right)=d_{S}\left(\mathcal{D}\left(V_{1}\right), l_{1}\right)=d_{S}\left(l_{0}, l_{1}\right)=1 .
$$

Hence, we have $i \geq 1$. Similarly, we have $p-i \geq 2$. These inequalities imply $p=i+(p-i) \geq 1+2=3$. Hence, $p=3$, and this implies that the Hempel distance of $V_{1} \cup_{f} V_{2}$ is 3 . Moreover, we have $i=1$, that is, $m_{1}=l_{1}$, since, if $i>1$, then $p-i<3-1=2$, a contradiction. 
To prove $m_{2}=l_{2}$, assume on the contrary that $m_{2} \neq l_{2}$. Then $m_{2}$, as well as $m_{1}\left(=l_{1}\right)$ and $m_{3}$, cuts $F_{2}$. By Lemma 2.2 and Claim 6.2 (3),

$$
\begin{aligned}
d_{F_{2}}\left(l_{1}, l_{3}\right) & =d_{F_{2}}\left(m_{1}, l_{3}\right) \\
& \leq \operatorname{diam}_{F_{2}}\left(m_{1}, \pi_{F_{2}}\left(m_{3}\right)\right)+\operatorname{diam}_{F_{2}}\left(\pi_{F_{2}}\left(m_{3}\right), l_{3}\right) \\
& \leq 4+4=8,
\end{aligned}
$$

which contradicts the inequality $d_{F_{2}}\left(l_{1}, l_{3}\right)>8$. Hence, $m_{2}=l_{2}$.

By Claim 6.2 (1), we have $m_{0}=l_{0}$ and $m_{3}=l_{3}$. Hence, $\left[l_{0}, l_{1}, l_{2}, l_{3}\right]$ is the unique geodesic realizing the Hempel distance.

Q.E.D.

\section{$\S$ Acknowledgement}

We would like to thank Dr Jesse Johnson for many helpful discussions, particularly for teaching us an idea of constructing a unique geodesic path in the curve complex.

\section{References}

[1] A. Abrams and S. Schleimer, Distances of Heegaard splittings, Geom. Topology, 9 (2005), 95-119.

[2] J. Berge and M. Scharlemann, Multiple genus 2 Heegaard splittings: a missed case, Algebraic and Geometric Topology, 11 (2011), 1781-1792.

[ 3 ] T. Evans, High distance Heegaard splittings of 3-manifolds, Topology Appl., 153 (2006), 2631-2647.

[ 4 ] W. J. Harvey, Boundary structure of the modular group. In Riemann surfaces and related topics, Proceedings of the 1978 Stony Brook Conference (State Univ. New York, Stony Brook, N.Y., 1978), pages 245-251, Princeton, N.J., 1981. Princeton Univ. Press.

[5] J. Hempel, 3-manifolds as viewed from the curve complex, Topology, 40 (2001), 631-657.

[6] A. Ido, Y. Jang and T. Kobayashi, Heegaard splittings of distance exactly n, Algebr. Geom. Topol., 14 (2014), 1395-1411.

[ 7 ] J. Johnson, Non-uniqueness of high distance Heegaard splittings, arXiv:1308.4599.

[8] T. Li, Images of the disk complex, Geom. Dedicata., 158 (2012), 121-136.

[9] H. Masur and Y. Minsky, Geometry of the complex of curves. I. Hyperbolicity, Invent. Math., 138 (1999), 103-149.

[10] H. Masur and Y. Minsky, Geometry of the complex of curves. II. Hierarchical structure, Geom. Funct. Anal., 10 (2000), 902-974.

[11] Ruifeng Qiu, Yanqing Zou and Qilong Guo, The Heegaard distances cover all nonnegative integers, Pacific J. Math., 275 (2015), 231-255.

[12] M. Yoshizawa, High distance Heegaard splittings via Dehn twists, Algebr. Geom. Topol., 14 (2014), 979-1004.

(A. Ido) Department of Mathematics Education, Aichi University of Education 1 Hirosawa, Igaya-cho, Kariya-shi, 448-8542, Japan

(Y. Jang, T. Kobayashi) Department of Mathematics, Nara Women's University

Kitauoya Nishimachi, Nara, 630-8506 Japan

E-mail address, A. Ido: ayakoido@auecc.aichi-edu.ac.jp

E-mail address, Y. Jang: yeonheejang@cc.nara-wu.ac.jp

E-mail address, T. Kobayashi: tsuyoshi@cc.nara-wu.ac.jp 